\title{
Representation of the Large Families Phenomenon in Social Networks
}

\author{
Bannykh G.A. \\ Institute of Public Administration and Entrepreneurship \\ Ural Federal University, Ural University of economics \\ Yekaterinburg, Russia \\ g.a.bannykh@urfu.ru
}

\author{
Kostina S.N. \\ Institute of Public Administration and Entrepreneurship \\ Ural Federal University \\ Yekaterinburg, Russia \\ s.n.kostina@urfu.ru
}

\begin{abstract}
The article discusses the reflection of the phenomenon of large families in social networks: through the representation of users in communities on the Vkontakte social network. The representation of modern universal phenomena in social networks reflects the trends of glocalisation. The purpose of this article is an attempt to describe representations of the phenomenon of a large family in a social network (using the example of the Vkontakte social network). Research methods analysis of the content of a social network (community, hashtags, comments, activity) Large families are represented in Vkontakte communities in a diverse and sufficient way, reflecting all its diversity in real practice. However, social practices are very limited - most of the publics and communities are self-help networks and NGO groups. The low level of representation of the problems of large families in the Vkontakte is associated with the age composition of the audience and the complex attitude of society towards large families.
\end{abstract}

Keywords—social networks; representation; large families; glocality; hashtag; like.

\section{INTRODUCTION}

Modern everyday life is represented in social practices that are increasingly happening in the virtual space. The characteristic of actual being and identity is called glocality as a universal phenomenon - the skill of perceiving the world as a global universal integrity while limiting its own actions in it.

Social networks as a form of new communication networks enhances the glocality of identity, generating multilevel localities: in the perception of stereotypes, world development scenarios, attitudes to universal phenomena, and so on (for example, J. Meyrowitz writes about the ability of social networks to make a person simultaneously present in different cultures and realities [1])

The family could be considered as a universal phenomenon and the most common form of the joint life of individuals. A large family is a family with many children, and locality forms different ideas about it in the minds and normative regulation of representatives of different cultures and countries: for example, in Russia a large family is considered to be a family in which three or more children are brought up, in the UK it is a family with four children, in Senegal - a family with seven or more children, etc.

The research interest is how the image of a large family appears on the social network, its daily social practices perceived by the rest of society and the large families themselves, in other words, how the phenomenon of large families is represented on the social network. Therefore, the purpose of this article is an attempt to describe the representations of the phenomenon of a large family in a social network (using the example of the Vkontakte social network). This network was chosen because of its prevalence among the population of Russia $(77 \%$ of the entire mobile audience in Russia use it, and the total monthly follow-up is 97 million [2])

\section{LITERATURE REVIEW}

The communicative space today is one of the main in which social phenomena are manifested. The most common communication option was social networks, which act as platforms for informing, interacting, and entertaining users. Social media are services and applications that allow users to interact with each other through the exchange of content (in the form of text, audio, photo and video). A social network is an interactive multi-user website with a number of mandatory qualities [3]: - users themselves are the creators of the content; - the site is an environment in which users interact with each other and create thematic groups; - users have access to communication functions with other users and social objects.

In the space of social networks, there is a representation of the phenomena of the real world and self-presentation of users. For J. Alexander, media information in accounts and groups on social networks is not only a symbolic representation of social interactions, but a configuration of experience, "windows into social reality" [4]. In the context of studying the phenomenon of large families, according to the idea of D. Boyd, profiles are presented on a social network that allow users to identify themselves with social groups[5]: the choice of a community associated with large families represents an attempt to identify a person with this social group. 
A visual representation in social media constructs a normatively idealised and aestheticised way of everyday life in accordance with expectations and socially approved patterns of behavior, stereotypes of vision and visual dominants adopted by social user groups. Representation means the operation of mediation and substitution, or the ability to represent someone or something through a combination of characters. However, representation does not at all strive for adequate reproduction; it creates a subjective reality. So, the image of large families represented on social networks can differ significantly from the real phenomenon, because it is represented through the actions and social practices of a certain group of users.

The social networks and practices of their users are the subject of interest of many Internet researchers. D. Boyd in his research defined the concept of "social networks" as web services that allow you to create a public or semi-accessible profile within a limited system, create a list of other users with whom you will be connected, and view a list of news contributed by others system users [5]. This definition of social networks allows you to look at them through the prism of social stratification and differentiation, highlighting the problem of user identity.

G. Reinhold introduces the concept of clusters communities that have appeared in new conditions, characterized by the arbitrary establishment of boundaries, determined situationally [6]. The individual is included in an unlimited number of such communities both offline and online, which allows us to talk about the cluster organization of the communicative and sociocultural space, which, in turn, leads to total control over the individual's choice of identification strategies and organization of personal daily life, especially in online mode.

K. Fuery suggests looking at "new media" as a new way of producing and interpreting texts, transforming ways of seeing [7], paying particular attention to discursive practices. J. Storey believes that more and more of our actions are associated with the use of media, and therefore we should think of them as part of everyday practices, without which the actions and interactions of people are simply unthinkable [8].

D. Miller studies the impact of social networks on people's daily lives. From the standpoint of anthropology, the researcher proves how diverse and diverse the use and influence of social networks are in the daily lives of various social categories of people. He introduces the concept of "multimedia", implying that the nature of the media does not determine the nature of their use [9].

In Russian studies of social networks, much attention is paid to the problems of interpersonal communications, various user practices and representation practices. E.S. Based on the analysis of personal profiles of users of social networks, E.S. Pronkina identified two opposing discursive strategies strategies for publicly telling about her life and strategies for building restrictions on access to her own representations [10]. Considerable attention is paid to research on virtual network communities. According to V.N. Popravko, the Internet community acts as a special form of being, a new type of social association [11].
R.V. Konchakovsky defining the Internet network community as interconnected individuals and / or social communities, the basis of socio-cultural unity of which is "community knowledge", divided them into global and local ones. social networks, in his opinion, relate to mass-contact local communities, characterized by fixed spatio-temporal boundaries of existence, entering as a structural element into a wider Internet community and formally limited by the scope of an electronic resource [12]

\section{RESEARCH METHODOLOGY}

In the theoretical part of the work, an analysis of documents and statistical data was carried out, which made it possible to judge about glocality and the possibilities of representation using social networks. The methodology was based on the phenomenology of everyday life, the theory of glocalisation of R. Robertson and J. Ritzer, and the theory of representation of J. Alexander. As part of an empirical study, we conducted an analysis of public groups on the social network Vkontakte, in the names of which there is a theme of large families, as well as hashtags and comments mentioning large families.

The social network Vkontakte presents three types of communities: public pages, groups and events. In the context of our analysis, we did not consider events and public pages that act more as marketing tools, rather than representing the desired phenomenon. Therefore, the object of analysis was precisely the groups, both open and closed, in the name of which the terms "large families", "large families", "large families", "large families" are reflected.

Research questions were as follows:

- How is a large social group represented on a social network;

- what social practices are available for representing the phenomenon of large families;

- how much quantitative data and qualitative indicators in a social network reflect real large families;

- How is the interaction of users within the communities according to the selected criterion.

\section{RESULTS}

For the keyword "large family", a search query revealed 1,434 thematic groups, "big family" - 1,026 groups. By the number of subscribers (participants), these groups are presented as follows: 95 groups with a total number of subscribers of more than 1000 people; 73 groups - from 500 to 1000 people; 96 groups - from 250 to 500 subscribers and 125 groups with the number of subscribers from 100 to 250 people. Of the indicated number, 1138 groups are registered precisely in Russia. Communities i.e. groups and official pages, but not events - 716, and all of them relate to the theme of "Family and Relations".

This is a fairly small number compared with groups on the theme of "family", of which there are more than fifty thousand. 
Of course, the prevalence of large families in the VK is not high. The question arises as to how much the represented groups represent Russian large families as a whole. Statistics show that the number of large families, that is, families with 3 children or more, is at the level of $5-5.5 \%$ of the total number of families; at the beginning of 2017, the number of large families of Russia was 1 million 566 thousand - in comparison with this, the participation rate in the largest community is 41 thousand people, that is, with the participation of at least one member of a large family, this is $2.6 \%$. Thus, the social network Vkontakte has not yet become for large families a phenomenon of everyday life or an urgent need. We will see further how problems, needs, and basic characteristics of large families are reflected in this network.

The mass fraction of all Vkontakte communities identified by the keyword "large families" is an association of large families in a certain territory - a region or a municipality (for example, "Association of Large Families of the City of Moscow", "Large Family of Mari El”, etc.). A significant part of this amount is made up of temporary groups dedicated to individual events (contests, meetings, concerts), as well as groups not marked as communities.

The largest group is a public of the Center for Assistance to Large Families, MnogoMama, with 41,361 members, the smallest is the Large Family (Show category) with 36 subscribers, and the Hard Life of a Large Father with 37 members.

A significant proportion of the total number of communities is made up of regional and municipal branches of one of the largest in Russia and the most widely represented in the social network Vkontakte, the NGO Center for Assistance to Large Families MnogoMama (20 communities in total). The protection of the rights and information on benefits for large families is much less dedicated to the number of groups about 7 .

An important element of the content analysis of thematic communities is the identification of "live" groups, that is, those in which there is a constant addition of content (relevant news, announcements and discussions appear). It should be noted that a significant proportion of the total number (109 or $16.3 \%$ ) are closed communities. They thus protect themselves from advertising, spam and other information that is not related to the topic of large families.

The largest number of groups are territorially assigned to Moscow and the Moscow Region - 52 communities. Among them can be identified groups uniting mothers and fathers, families with twins and weathers, Orthodox large families, online media "Multamama TV", business blogs of large mothers, many support groups for solving basic issues of large families, needlework, beauty, choir, etc. . Only 7 groups are related to the topic "Relations and Family".

The largest group, MnogoMama, is a non-profit organization with a central office in Moscow and several branches and representative offices both in Russia and abroad. The organization provides assistance to more than 75,000 large families throughout Russia, 40,000 of them in Moscow and the Moscow Region. Mnogomamama has a group on
VKontakte, a Facebook page, her own website and a TV channel (on YouTube). The group is quite active - 4-8 publications a day appear in it, mainly posts about events, events and various measures for large families. The activity of the subscribers themselves depends on the message - some receive 400-450 views, some actively comment on photo content.

In St. Petersburg, there were 98 groups with a name that included the term "large families", 22 groups were related to the subject of "Relations, family." The largest group is the Large families of St. Petersburg and the Leningrad Region with 9,078 members. The activity in the group is high - from 3 to 10 posts are published daily with topics covering current issues of the life of large families: from regulatory acts to lafhacks and contests. The most active users under posts with various questions and advice on measures of state support (the use of social cards, accrual of benefits, contacting doctors and the clinic).

The second largest open group "Large families of the Leningrad Region" with 6802 participants is also active, but is not used to distribute official information.

The behavior of group subscribers on the topic of large families and the content of such groups differ regionally. So, the most talked about are publications in the public of St. Petersburg and Yaroslavl, the most viewed are the publications of groups from Moscow, Yaroslavl and Tyumen, the subscribers of these groups are most active in "likes" and reposts.

According to the results of the analysis, the activity according to the days of the week and time of day is not uniform among users participating in groups. So, at the largest public - the Help Center in Moscow, the activity of participants rises after 18-00 and lasts until 22-00 hours on Mondays and Sundays mainly. The participants of the group of large families of St. Petersburg - the activity is highest from $22-00$ to 2 hours in the morning and from 6 to 8 in the morning (on Fridays, Saturdays and Sundays). The participants of the public Joy from Tyumen - at 0-00 at night and from 5 to 8 in the morning (on Sundays).

In the public group of large families of Yaroslavl - at 6 in the morning and at 17-00 (on Wednesdays and Saturdays). In large families from Buryatia, the greatest activity is manifested at 6 a.m. on Tuesdays and Sundays. This specificity is determined by the status of the organizers and administrators of the groups themselves: in those groups that reflect the activities of organizations it can be weekdays and weekends, for a public volunteer - this time is limited by free time.

Below are the data on the main indicators of the activity of the selected groups on the Vkontakte network (See Tab 1). 
courageous, of course, the main quality of all families is decency and mutual assistance. The most important thing in this world is not to get lost, to communicate, to exchange experience, not to become locked in your problems "(analysis is impossible to carry out, since the group is closed). The remaining groups are dedicated to specific families and consist of 20-25 people.

There are 2 open groups in the city of Krasnoyarsk, 3 groups in the city of Perm, 2 of them are closed, all of them are dedicated to specific families. In 1 group, it was revealed in such cities as Surgut, Podolsk, Voronezh, Kazan, Krasnodar, Ufa, Chelyabinsk, etc. Moreover, a significant part of them is closed.

The next direction of content analysis of social networks is the analysis of hashtags, user comments of various news, articles and announcements. Currently, Vkontakte has more than 934 thousand comments and user entries with the words "large families" and "big families".

For analysis, we developed a classifier of keywords, which were divided into positive, negative and neutral by emotional coloring. The words "joy", "happiness", "well-being" and others were attributed to positive ones. Negative were "need", "trouble", "poverty", "illness", etc. The neutral group included the words: "help", "benefits", "discounts", etc.

The second keyword that was used to analyze Vkontakte groups is "Big Family". For this keyword, the largest group is the "Family with a capital" group with 7,494 members, followed by the online media "More Life!" Information portal for the families of the Russian Federation "with 6,014 subscribers, in third place -" Big Seven "things for free / Togliatti exchange" with 5,171 participants.

Among the communities on the topic "Family and Relationships", 61 groups were found by keywords, the largest of which is the Big SevenJ group in the city of Surgut (Khanty-Mansi Autonomous Okrug-Yugra) with 2,021 members. The group has an active life - up to 5 posts per week, the active use of hashtags \#manyChildrenTogether \#combineRussiawithmanychildren and from 450 to 500 views per post. The next group is from Podolsk with 1820 subscribers. The group is even more active - more than 5 posts per week, more than 140 views each, tags are also actively used.

In a territorial context, the search yielded the following results. In Moscow, 1 group was found - "Big Family. Russia "with 1019 participants. In this group, an average of 1 publication per day, which accounts for 120 views, 1-5 reposts, 9-89 likes, a sufficient number of reposts with information from the online media "More Life!" | Information portal of the families of the Russian Federation "

In the city of St. Petersburg, 7 groups were found, the most numerous of which was "Large and simply large friendly families" with 45 participants. The description of the information contains the following motto: "I really want our children to not be looked upon with contempt in our society, we must prove to everyone that our families are no worse than others, that we can learn ourselves and teach our children that we can also do business no worse that we are united,
TABLE II. FREQUENCY OF USING KEYWORDS IN COMMENTS ON LARGE FAMILIES ON THE VKONTAKTE

\begin{tabular}{|c|c|c|c|c|c|}
\hline \multicolumn{2}{|c|}{ Positive words } & \multicolumn{2}{|c|}{ Negative words } & \multicolumn{2}{|c|}{ Neutral words } \\
\hline $\begin{array}{c}\text { Happin } \\
\text { ess }\end{array}$ & 11796 & $\begin{array}{c}\text { Disable } \\
\mathrm{d}\end{array}$ & 36818 & Help & 66200 \\
\hline Joy & 8366 & $\begin{array}{c}\text { Povert } \\
\mathrm{y}\end{array}$ & 8685 & $\begin{array}{c}\text { Privilege } \\
\mathrm{S}\end{array}$ & 48684 \\
\hline $\begin{array}{c}\text { Succes } \\
\mathrm{S}\end{array}$ & 4008 & $\begin{array}{c}\text { Troubl } \\
\mathrm{e}\end{array}$ & 4558 & $\begin{array}{c}\text { Discount } \\
\mathrm{s}\end{array}$ & 29251 \\
\hline Wealth & 2548 & $\begin{array}{c}\text { Diseas } \\
\mathrm{e}\end{array}$ & 4514 & Support & 22079 \\
\hline \multirow[t]{3}{*}{$\begin{array}{c}\text { Vocati } \\
\text { on }\end{array}$} & 417 & $\begin{array}{c}\text { Povert } \\
y\end{array}$ & 3214 & Gifts & 18300 \\
\hline & & $\begin{array}{c}\text { Misfort } \\
\text { une }\end{array}$ & 699 & Mortgage & 9753 \\
\hline & & & & $\begin{array}{c}\text { Maternal } \\
\text { capital }\end{array}$ & 5281 \\
\hline
\end{tabular}

For all the time in Vkontakte, 18108 entries were made with the hashtag \#LargeFamily, which received almost 8 million views and more than 21 thousand comments (See Table III). We can state a fairly high activity of network users associated with this hashtag.

Searching for the hashtag \#Bigfamily yielded the following results: in total, 104 entries with 50,000 views and 241 comments were accompanied by this hashtag. Compared to the previous tag, this one is much less popular and much less attached to the topic of large families. 
In terms of the number of reposts, the publication of the Head of the Republic of Tuva on July 8, 2016 on the decision to support large families with free coal in winter (at least 5 children) also became the most active. In the publication, the Chapter says that having many children is a special unique tradition of the residents of the republic: "There have always been many children in the Tuvan family - at least 10! ". 123 reposts and 528 likes were made on it.

\section{CONCLUSION}

By the number of likes, the most popular publication was made on January 9, 2019 by the Head of the Republic of Tuva Sholban Valerievich Kara-ool on his own VKontakte page. It is dedicated to the situation with a mother with many children, who lost her husband, expects a sixth child and supports her elderly parents, and at the beginning of the year her house burned down. A woman works as a teacher. The head of the republic described her situation and decided to apply for her to be awarded. The publication has collected 19 thousand views and 1276 likes.

Within the considered groups, the most popular by the number of likes was the publication in the public of Multinam from 07/17/2017 about the photo contest. 1639 likes and 441 reposts were made for publication.

The second most popular publication is from the public publication "Large Families of Yaroslavl" dated April 11, 2019 about a boy from an orphanage who asks to decide his fate. 1418 likes and 576 reposts were made for publication.

The third most popular publication is also from the publication "Large Families of Yaroslavl" dated October 31, 2018 , devoted to the topic of younger schoolchildren who are lost and died.

By the number of comments, the most active is the publication in the community "Large Families of St. Petersburg and the Leningrad Region" dated March 24, 2018 with the text "Girls who are not difficult to discard please lawsuits I I want to apply for alimony for myself and my children while I am married. " 577 responses were received for publication. Users in the process communicated with each other, gave tips, links, recommendations.

The second most commentary publication was also made in this group $07 / 13 / 2018$. "Girls who registered a disability in a child with ZPR, do you really need to be registered with a psychiatrist for 1 year to get it?" And received 512 comments.

Also, according to the number of comments, one of the most popular posts was made in the Country FM group of June 8,2016. The basic idea is "Let's try to calculate the ideal number of children in a family!" How many children do you want and how many do you have now, how do you deal with them? "307 user comments were received. Most of the answers are simple user communication ("hello, N!", "Glad to see you, J!"), The answers are essentially 198. The message generated active user communication on the topic of the family and the number of her children. The idea of having many children was supported by about $40 \%$ of users, relying on the fact that they themselves were brought up in a family with brothers and sisters.
Analysis of the content of the social network Vkontakte showed that the problems of large families presented in it do not reflect the prevalence of this phenomenon in Russian society. The largest groups in the VK associated with large families were created by non-profit organizations and are located in the central regions of the Russian Federation Moscow and St. Petersburg. In other regions, the activity of large families in the $\mathrm{VC}$ is much lower, with the exception of those territories where large NPOs are associated with solving the problems of large families.

Most often, VC is used as a way to solve the problems of large families - both by individual families and by territorial communities. Those. in fact, VK is used as a social support network. From this perspective, it is quite natural that the greatest response from the audience is caused by "problem" posts, including those related to negative events in the life of large families. As in previous studies of the authors on the topic of large families, it can be concluded that information is not very much needed from large families and for large families $[13,14]$.

The low level of representation of the problems of large families in the VK is, in our opinion, associated with the following reasons. Firstly, this is the age composition of VK users - on the one hand, the main audience of VK - young people - is weakly interested in this issue, on the other hand, the largest part of large families is made up of a more "adult" audience.

Secondly, this is a reluctant representation of their lives and problems by large families themselves, which is caused, among other things, by a rather complicated attitude in society towards large families, when often large families are perceived as "abnormal", asocial, "dependents"

\section{References}

[1] J. Meyrowitz "The rise of glocality: New senses of place and identity in the global village". In: K. Nyíri (ed.), The global and the local in mobile communication, Passagen Verlag, Vienna. 2005.

[2] Social network users in Russia: statistics and audience portraits. URL: https://vk.com/@cerebro_vk-polzovateli-socsetei-v-rossii-statistika-iportrety-auditori).

[3] V. D. Vinnik Social networks as a phenomenon of the organization of society: essence and approaches to use and monitoring // Philosophy of Science. 2012, 4, pp. 110-126

[4] J. Alexander The Meanings of Social Life: Cultural Sociology Oxford University Press; 1 edition (November 24). 2005, pp. 296

[5] D. Boyd Social media: A phenomenon to be analyzed. Social Media + Society 1-2, 1-3.

[6] G. Reinhold Smart Crowd: A New Social Revolution, Moscow: FairPress. 2006, 416. 
[12] R.V. Konchakovsky Network Internet community as a sociocultural phenomenon. Candidate's thesis. Yekaterinburg. 2010, 25.

[7] K. Fuery New Media. Culture and Image. New York : Palgrave Macmillan. 2009, 173.

[8] J. Storey Mediatized Spaces of Intimacy Culture, Space and Power: Blurred Lines, Maryland : Lexington Books. 2015, pp. 101-114

[9] D. Miller "Tales From Facebook" Cambridge. PolityPress. 2011, 218.

[10] E.S. Pronkina Representations of Love Life in Social Media (By the Example of the Social Networking Service "VKontakte"). Observatory of Culture. 2017, 14(4), pp. 404-410.

[11] V. N. Popravko internet community: specificity and role in the formation of social space. Abstract of dissertation for the degree of candidate of philosophical sciences. Tomsk. 2011, 24.

[13] Alexsandr S. Kuznetsov. Russian Professor's meeting. Russian Journal of Physical Education and Sport. 2019, 14(1), pp. 17-22. DOI: 10.14526/2070-4798-2019-14-1-18-24

[14] G A Bannykh, E V Zaitseva, S N Kostina, A I Kuzmin. Image of a mother of many children in the space of the russian internet. Society and power. 2019, 1 (75), pp. 42-52. 